\title{
The pion polarisability and more measurements on chiral dynamics at COMPASS
}

\author{
Jan Michael Friedrich*† \\ on behalf of the COMPASS collaboration \\ Physik-Department, Technische Universität München \\ E-mail: jan@tum.de
}

\begin{abstract}
Within the physics program of the COMPASS experiment at CERN pion-photon reactions are measured via the Primakoff effect, referring to processes in which high-energetic pions react with the quasi-real photon field that surrounds the target nuclei.

The production of a single hard photon in such a pion scattering at lowest momentum transfer to the nucleus is related to pion Compton scattering. From the measured cross-section shape, the pion polarisability has been determined, a result that has been published meanwhile as a Physical Review Letter [1]. The COMPASS measurement is in tension with the earlier dedicated measurements, and rather in agreement with the theoretical expectation from chiral perturbation theory. The analysis of a more recent high-statistics data taking is underway.

Reactions with neutral and more charged pions in the final state are measured and analyzed as well. At low energy in the pion-photon centre-of-momentum system, these reactions are governed by chiral dynamics and contain information relevant for chiral perturbation theory. At higher energies, resonances are produced and their radiative coupling is investigated.
\end{abstract}

The 8th International Workshop on Chiral Dynamics, CD2015 ***

29 June 2015 - 03 July 2015

Pisa, Italy

\footnotetext{
* Speaker.

$\dagger$ supported by the German ministry BMBF, the Maier-Leibnitz-Labor der LMU und TU München, and the DFG Cluster of Excellence "Origin and Structure of the Universe"
} 


\section{Pion-photon reactions as test of chiral perturbation theory}

Properties of the pions $\left(\pi^{-}, \pi^{0}, \pi^{+}\right)$are of crucial interest in understanding quantum chromodynamics (QCD), since the pion is the lightest system with confinement of quarks and gluons by the strong force. The pions are identified in the framework of the low-momentum expansion of QCD, chiral perturbation theory (ChPT) as the Goldstone bosons emerging from the spontaneous breaking of chiral symmetry. For more details of this aspect, see the contribution of Gerhard Ecker in these proceedings [2].

Pion-pion scattering has been studied by several approaches, e.g. in kaon decays, and successfully described within ChPT. In contrast, for pion-photon interactions even the most fundamental process of Compton scattering has remained a riddle for the past 30 years: The leading structuredependent term in this process is the polarisability, and its extraction from the first experimental data in 1983, confirmed by some later experiments, while deviating from other results, as discussed in more detail in Sec. 5. Clarifying this subject was the prime motivation for the experimental work presented here. On top of this, other pion-photon interactions with more pions in the final state came into reach, and are studied as well. This is, on the one hand, a research subject by itself, on the other hand, it represents a powerful check of the common aspects in the employed experimental techniques.

\section{Primakoff technique}

In 1951, Primakoff proposed [3] to make use of the intense electric field in the proximity of nuclei, which can be treated in a high-relativistic reference frame as a source of quasi-real photons, to study interactions with strongly-interacting particles. The original idea concerned the measurement of the $\pi^{0}$ lifetime by photon-photon fusion, but it was later realized that interactions of high-energetic hadrons with the nuclear Coulomb field represent similarly a scattering off the quasi-real photon density, and consequently the whole class of such hadron interactions is referred to as Primakoff reactions. The process is depicted in Fig. 1. The main contribution comes from impact parameters of the pions of a few nuclear radii, where the electric field is still as strong as several $100 \mathrm{kV} / \mathrm{fm}$. This displays how even a small polarisability as it is expected for hadrons, can be measured as a modification of the cross-section for bremsstrahlung emission. The crosssection formula for a Primakoff reaction $\pi^{-} A \rightarrow X^{-} A$ on a nucleus $A$ reads, in one-photon exchange approximation (see e.g. [4]),

$$
\frac{d \sigma}{d s d Q^{2} d \Phi}=\frac{\alpha}{\pi\left(s-m_{\pi}^{2}\right)} \cdot F_{\text {eff }}^{2}\left(Q^{2}\right) \cdot \frac{Q^{2}-Q_{\min }^{2}}{Q^{4}} \cdot \frac{d \sigma_{\pi \gamma}}{d \Phi}
$$

and bases on the factorization into the quasi-real photon density multiplying the cross-section $d \sigma_{\pi \gamma} / d \Phi$ for the real-photon subprocess $\pi^{-} \gamma \rightarrow X^{-}$, with $d \Phi$ being a proper phase space parameterization for the final state of the subprocess. Mandelstam- $s$ is the squared total energy in the $\pi^{-} \gamma$ subsystem, $Q^{2}$ is the momentum transfer to the nucleus $A, \alpha \simeq 1 / 137$ is the fine structure constant, $m_{\pi}$ the rest mass of the charged pion, $Q_{\min }=\left(s-m_{\pi}^{2}\right) / 2 p$ is the minimum momentum transfer for given $s$ and beam momentum $p . F_{\text {eff }}^{2}\left(Q^{2}\right)$ is the form factor of the target nucleus with charge $Z$. It is $F_{\text {eff }}^{2}\left(Q^{2}\right) \approx Z^{2}$ when $Q^{2} \ll 10^{-3} \mathrm{GeV}^{2} / c^{2}$ and in addition $s$ is sufficiently small, such 


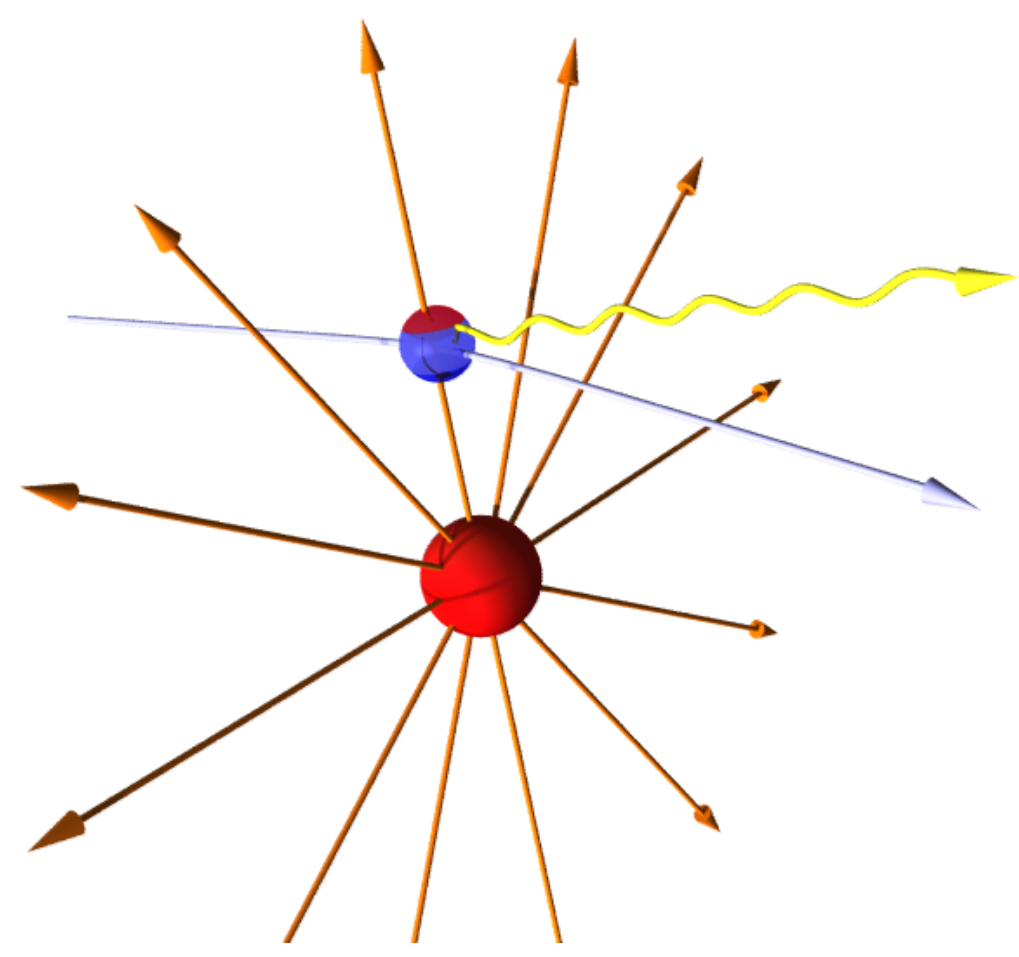

Figure 1: Visualization of the Primakoff Compton process: A high-energetic pion scatters in the electric field of a nucleus. For the magnetic contribution, it is to be realized that the nucleus passing the pion at high velocity represents an electric current inducing a magnetic field at the position of the pion.

that the longitudinal compontent $Q_{L}$ (given in size approximately by $Q_{\min }$ ) is negligible. For the first process of interest here, the final-state $X^{-}$is again $\pi^{-} \gamma$ such that the involved subprocess is pion Compton scattering $\pi^{-} \gamma \rightarrow \pi^{-} \gamma$. The respective cross-section reads

$$
\begin{aligned}
\frac{d \sigma_{\pi \gamma}}{d \Omega_{c m}}= & \frac{\alpha^{2}\left(s^{2} z_{+}^{2}+m_{\pi}^{4} z_{-}^{2}\right)}{s\left(s z_{+}+m_{\pi}^{2} z_{-}\right)^{2}}-\frac{\alpha m_{\pi}^{3}\left(s-m_{\pi}^{2}\right)^{2}}{4 s^{2}\left(s z_{+}+m_{\pi}^{2} z_{-}\right)} . \\
& \cdot\left(z_{-}^{2}\left(\alpha_{\pi}-\beta_{\pi}\right)+\frac{s^{2}}{m_{\pi}^{4}} z_{+}^{2}\left(\alpha_{\pi}+\beta_{\pi}\right)-\frac{\left(s-m_{\pi}^{2}\right)^{2}}{24 s} z_{-}^{3}\left(\alpha_{2}-\beta_{2}\right)\right)
\end{aligned}
$$

where $z_{ \pm}=1 \pm \cos \theta_{c m}$ and $\theta_{c m}$ is the scattering angle in the $\pi^{-} \gamma$ center-of-momentum system, and the pion structure enters through the electric and magnetic polarisabilities $\alpha_{\pi}$ and $\beta_{\pi}$. The solid angle $d \Omega_{c m}=d \cos \theta_{c m} d \varphi_{c m}$ is the concrete form that the phase space $d \Phi$ of Eq. (2.1) takes on for the considered two-body reaction at fixed $s$. In the following, the sum $\alpha_{\pi}+\beta_{\pi}$ which is expected to be small, and also the influence of the quadrupole polarisabilities $\alpha_{2}$ and $\beta_{2}$ is neglected. Then, the relative effect of the polarisability $\alpha_{\pi}=-\beta_{\pi}$ on the cross-section, Eq. 2.1, integrated in the small-momentum transfer region $Q^{2} \leq 10^{-3} \mathrm{GeV}^{2} / c^{2}$ and depending only on the fraction of energy transfered from the incoming pion beam to the emitted photon, $x_{\gamma}=E_{\gamma} / E_{\text {beam }}$, can be simplified into

$$
R=\frac{\sigma\left(x_{\gamma}\right)}{\sigma_{\alpha_{\pi}=0}\left(x_{\gamma}\right)}=1-\frac{3}{2} \cdot \frac{m_{\pi}^{3}}{\alpha} \cdot \frac{x_{\gamma}^{2}}{1-x_{\gamma}} \alpha_{\pi} .
$$


This relation is used to extract the polarisability from the measurement of the photon energy spectrum in the the Primakoff process $\pi^{-} Z \rightarrow \pi^{-} \gamma Z$ on a nucleus with charge $Z$, as it has been done in the first measurement of this kind at Serpukhov [5].

As can be read off Eq. (2.1), the cross-section for Primakoff reactions is proportional to the square of the nuclei charge $Z^{2}$. Since the competing strong interactions only grow roughly with $Z^{2 / 3}$, one the Primakoff contribution can be enhanced by choosing high- $Z$ targets. For systematic effects discussed later, very heavy targets are also disfavored, and for the experiment described here, a target with medium-heavy nickel nuclei was chosen. Measuring with different target nuclei in the same setup allows to study the Z-dependence of the different contributions.

\section{Pion polarisability measurement at COMPASS}

The COMPASS experiment deploys secondary hadron and tertiary muon beams from the CERN $450 \mathrm{GeV}$ super proton synchrotron (SPS). Its multi-purpose detector concept allows for a wide range of investigations in hadron physics, with high-precision and high-rate capable tracking, particle identification and calorimetry in both stages of the magnetic spectrometer. The layout

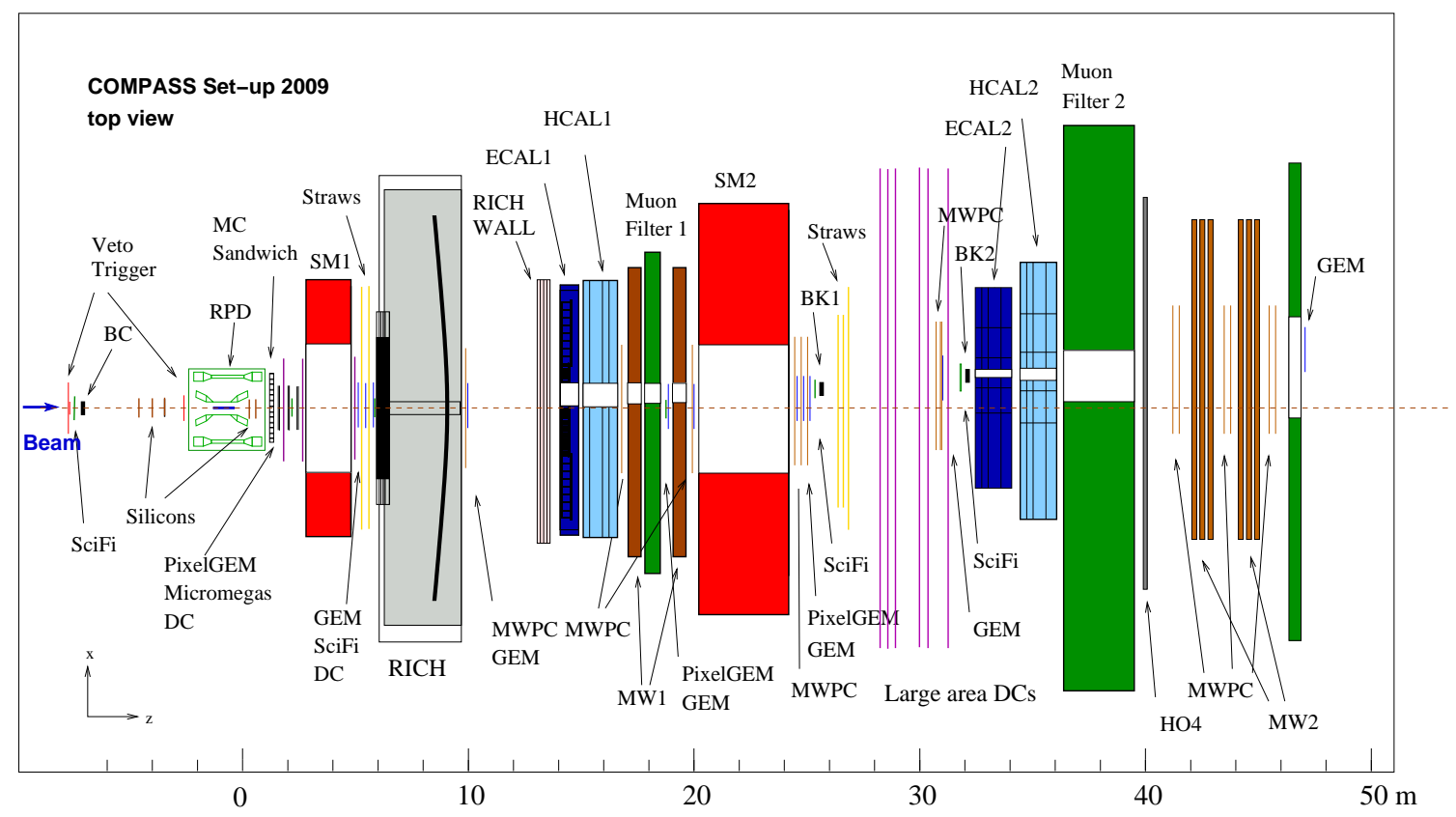

Figure 2: Layout of the COMPASS setup for hadron beam as used in the beam times of 2008 and 2009. A detailed description of the employed detectors is found e.g. in Reference [21]. The spectrometer magnets SM1 and SM2, surrounded by tracking detectors and followed by the calorimeters, constitute the two stages of the setup.

of the setup is shown in Fig. 2. The two stages are optimized for low and high momentum particles, respectively, and allow a momentum determination of better than $1 \%$ in a wide range, from about $1 \mathrm{GeV}$ up to the beam momentum in the range of $200 \mathrm{GeV}$.

The measurement of the pion polarisability has been one of the original goals of the proposal for the COMPASS experiment. After a pilot run in the year 2004, the data presented in the following have been collected in a two-week beam time in 2009, with significant improvements in the 
calorimetry and the trigger system which based on the detailed analysis of the 2004 data. One of the conclusions along with preparing the data taking in 2009 [6] was that lead is not a favorable target material despite the high nuclear charge $Z$, since the radiative corrections due to multiple photon exchange and screening are large and represent a non-negligible source of systematic uncertainty. Consequently, the measurement was performed with a $4 \mathrm{~mm}$ thick nickel disk as nuclear target. A very thin tungsten target, placed $40 \mathrm{~cm}$ downstream of the main target, allows to confirm the expected $Z^{2}$-dependence of the Primakoff contribution, however the achieved event sample on the tungsten target is not sufficient for the detailed polarisability analysis. The measurement and the extraction of the pion polarisability signal is described in detail in [1].
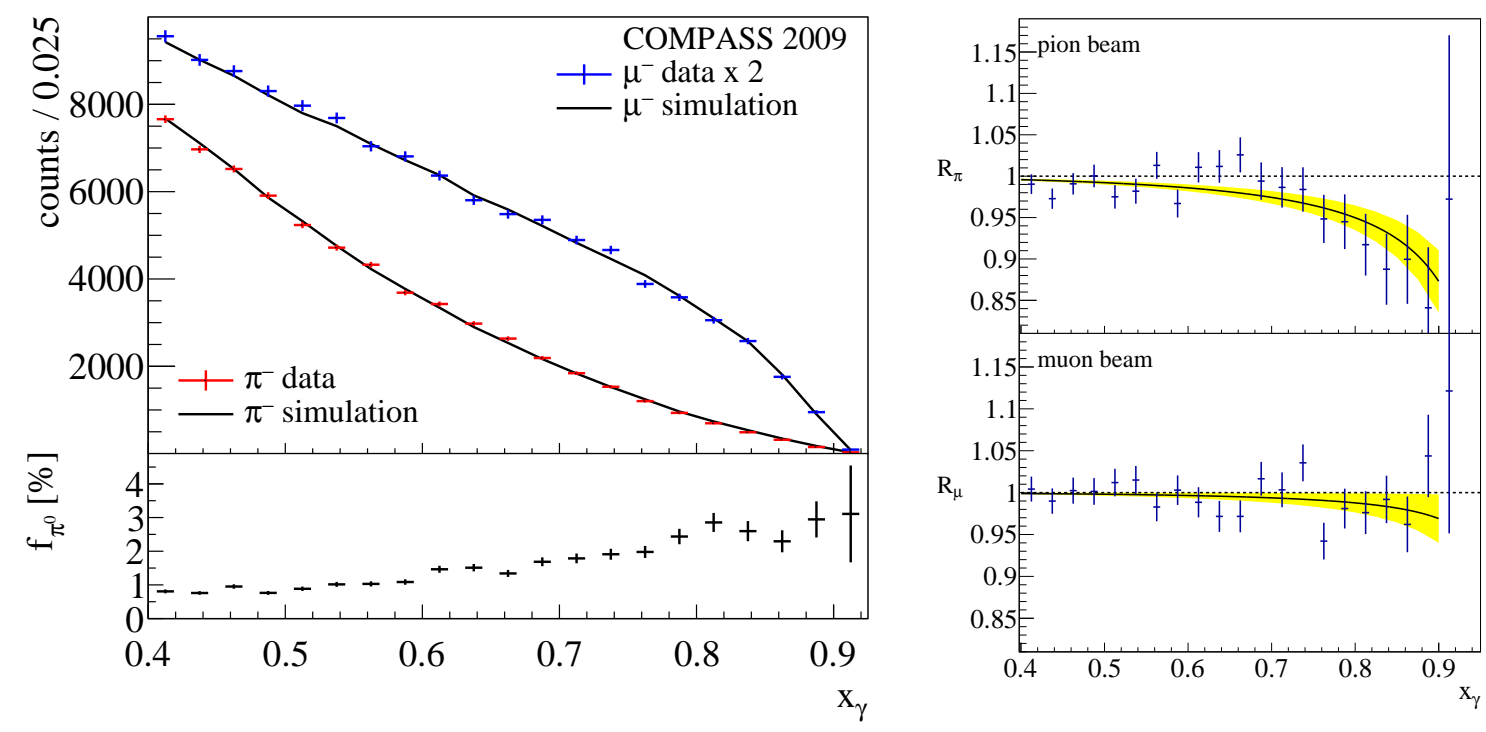

Figure 3: Determination of the pion polarisability through the process $\pi^{-} \mathrm{Ni} \rightarrow \pi^{-} \gamma \mathrm{Ni}$, and control measurement with muons $\mu^{-} \mathrm{Ni} \rightarrow \mu^{-} \gamma \mathrm{Ni}$. On the left, the spectra in the variable $x_{\gamma}$ are shown, together with the respective expectation from the simulation. From the data taken with pion beam, the background from $p i^{0}$ events has to be subtracted, whose fraction is shown in the lower panel. On the right plots, the ratio between the measured spectrum and the expectation without polarisability effect is shown. The curves display the fits according to equation 2.3. Figures from [1].

The result of the measurement is shown in Fig. 3. For the determination of the polarisability, the photon energy spectrum is examined according to Eq. 2.3. In the case of muon beam, the shape of the distribution is in excellent agreement with the simulation as shown in the upper graphs of Fig. 3. The size of the "false polarisability" signal, in agreement with zero within the fit uncertainty, of $\pm 0.5 \cdot 10^{-4} \mathrm{fm}^{3}$ is taken as an estimate for apparative imperfections not described by the simulation, e.g. concerning the tracking.

In the case of pion beam, the experimental spectrum has been corrected for the background estimation given in Fig. 3, left lower plot, and is divided by the simulation, in which the bremsstrahlung cross-section for a pointlike spin- 0 boson has been taken. The result of this procedure is shown in the lower graphs of Fig. 3. By fitting the distribution according to Eq. 2.3, the pion polarisability is determined from the COMPASS 2009 data to be $\alpha_{\pi}=\left(2.0 \pm 0.6_{\text {stat }}\right) \cdot 10^{-4} \mathrm{fm}^{3}$.

Radiative corrections have been applied on the level of the simulation event-wise, starting 

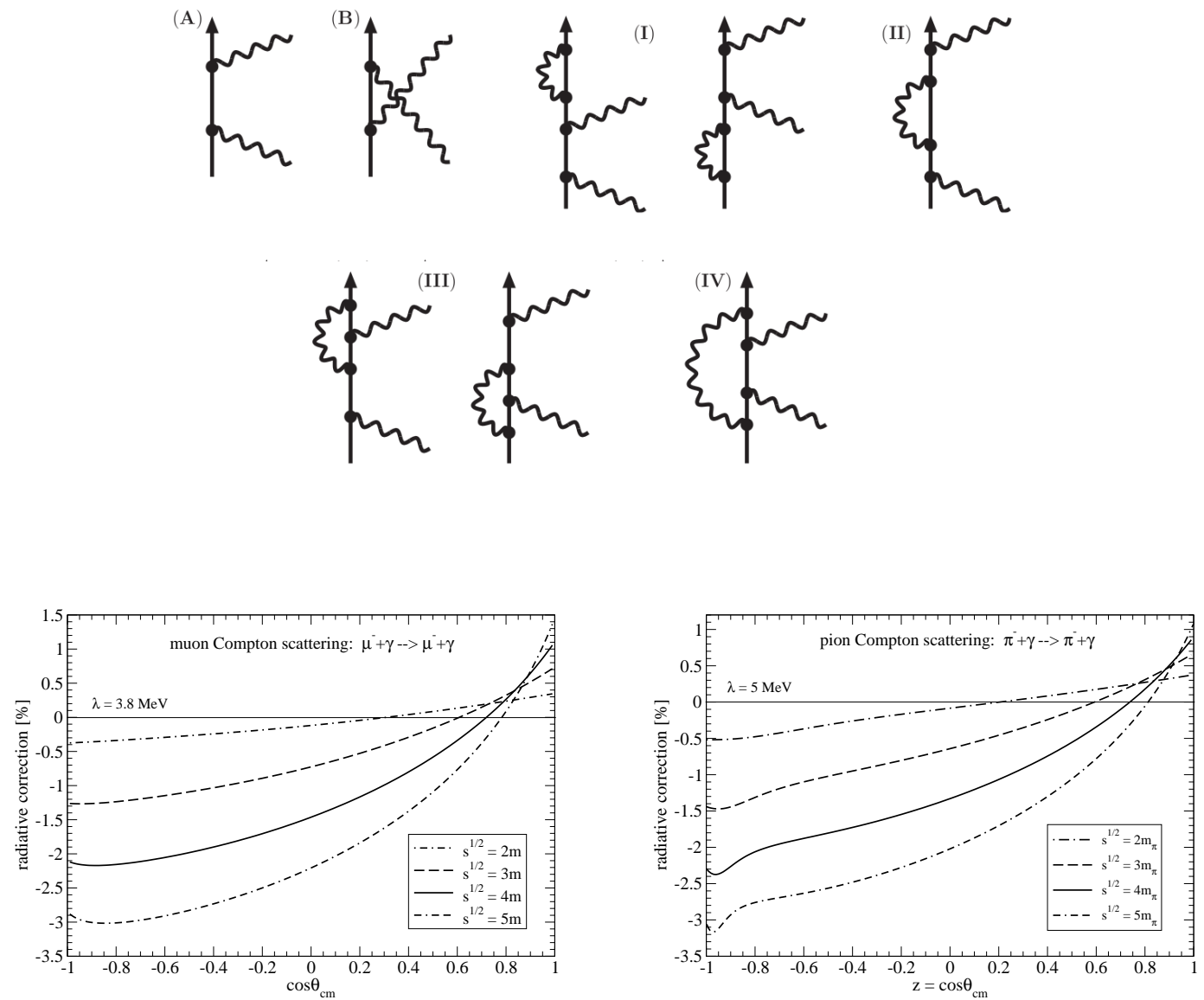

Figure 4: Electromagnetic radiative corrections for muon and pion Compton scattering. Examplarily, the Feynman graphs for the virtual corrections in the muon are shown (top part of the figure). For the pion, there are more graphs due to the additional point-couplings in case of a spin- 0 particle [7]. The lower graphs (from [7, 8]) show the radiative corrections, to be employed as multiplicative factor to the non-radiative process, for different $\mathrm{CM}$-energies in the region of interest for the pion polarisability measurement. For a full discussion see [9].

from the published calculations $[7,8]$ for the case of pion and muon Compton scattering, respectively, and extrapolating to the Primakoff kinematics at $Q^{2} \neq 0$. The small difference between the corrections for pion and muon stems from their spin- 0 and spin- $\frac{1}{2}$ nature, respectively. The error involved in the approximation $Q^{2} \approx 0$ has been shown to be below $10 \%$ of the correction itself [6] and thus negligible. Along with the uncertainty of the vacuum polarisation and multiple-photon exchange corrections, the uncertainty of the radiative corrections to the determination of the pion polarisability has been estimated to be about $\pm 0.3 \cdot 10^{-4} \mathrm{fm}^{3}$, and this value is included in the list of systematic uncertainties.

An additional background contained in the data stems from scattering of the beam particles off the electrons in the target. Since the recoiling electrons may lose practically all their energy by bremsstrahlung, this leads to a signature very similar to the intended process of photon emission when scattering off the nulcei. The contribution of this process has been investigated, and its 
impact on the polarisability determination included as systematic uncertainty of $\pm 0.2 \cdot 10^{-4} \mathrm{fm}^{3}$ on the polarisability signal.

Summing all discussed systematic uncertainty contributions leads to a total of $\pm 0.8 \cdot 10^{-4} \mathrm{fm}^{3}$. So, the COMPASS result for the pion polarisability from the 2009 data [1] is

$$
\alpha_{\pi}=\left(2.0 \pm 0.6_{\text {stat }} \pm 0.7_{\text {sys }}\right) \cdot 10^{-4} \mathrm{fm}^{3} .
$$

\section{Higher-order correction: One-loop chiral calculation versus dispersion relations}

The experimental data have been corrected for the higher-order correction as expected from the one-pion loop calculation in ChPT (cf. e.g. [4]). An alternative way to treat the higher-order influence is the employment of dispersion relations, the most modern evaluation given in $[15,16]$. As it was raised as a possible critique, in how far the two approaches may vary for pion Compton scattering, while it was shown for $\gamma \gamma \rightarrow \pi \pi$ that there is no conflict between ChPT and dispersion theory $[15,16]$. Fig. 5 shows that in the kinematic region of interest, there is agreement between

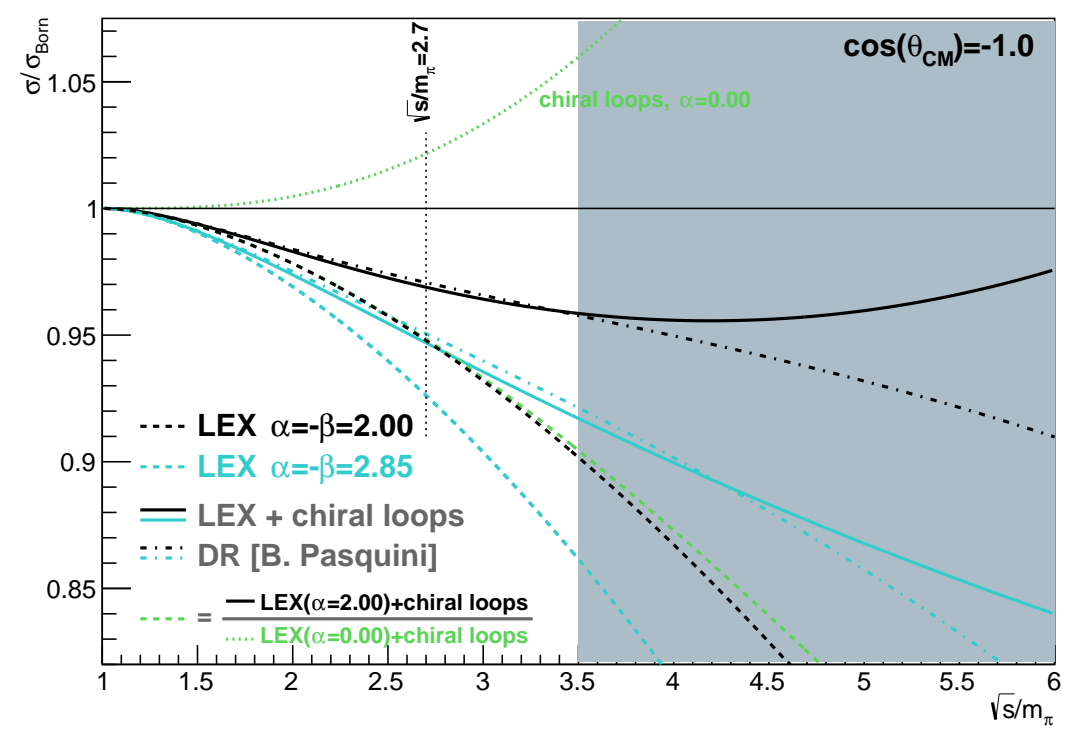

Figure 5: Comparison of the effects of the pion polarisability and higher orders on the cross-section of the process $\pi^{-} \gamma \rightarrow \pi^{-} \gamma$ for backward scattering. Black lines refer to a pion polarisability value $\alpha=2.0$. $10^{-4} \mathrm{fm}^{3}$, light-blue lines to a value $\alpha=2.85 \cdot 10^{-4} \mathrm{fm}^{3}$. Dashed curves show the effect of the polarisability alone, while the contrinuous and dash-dotted curves include the next order from one-pion loop calculation and dispersion relations, respectively. The COMPASS data are centered around the indicated value $\sqrt{s}=$ $2.7 m_{\pi}$, the region cut by the condition $m_{\pi \gamma}<3.5 m_{\pi}$ is grey shaded. Dispersion relation calculation courtesy B. Pasquini.

the ChPT one-loop calculation and dispersion relations, kindly provided by B. Pasquini [17], on the permille level. 


\section{Discussion of the pion polarisability result}

The presented preliminary COMPASS value for the pion polarisability is compared to previous experimental results in Fig. 6. Historically (left graph) the first result obtained at Serpukhov [5] had been confirmed much later by the dedicated experiment on radiative pion photoproduction at MAMI [10]. In the mean time, the available data on $\gamma \gamma \rightarrow \pi^{+} \pi^{-}$at $e^{-} e^{+}$-colliders were reinterpreted by several authors $[11,12,13,14]$ claiming very different values for the pion polarisability, inspired by the assumptions on pion dynamics and the related low-energy constants that enter in this interpretation. In that regard, the COMPASS result is in significant tension with the
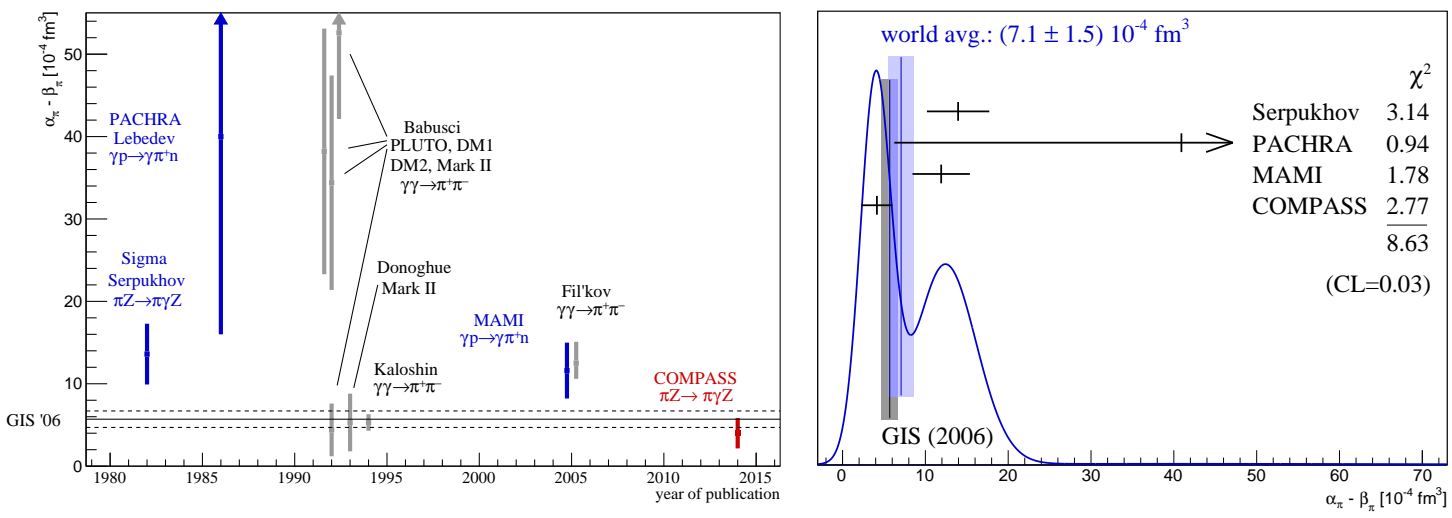

Figure 6: Placement of the preliminary COMPASS result on the pion polarisability in the world data (left) and the ideogram in "PDG style" [19] (right). Plots are from [20], where also a full discussion of the experimental data is found. The symbol GIS06 refers to the theoretical prediction as given in Reference [18].

earlier experimental determinations of the pion polarisability, as the ideogram representation in Fig. 6 (right) shows, where only the dedicated experiments for the pion polarisability are included. Instead, the new result is found in good agreement with the expectation of chiral perturbation theory [18].

In view of the small value obtained for the pion polarisability in this analysis, it is of high interest what the data taken in the year 2012 at COMPASS with a very similar setup as described here will show. For this data set, the statistical uncertainty is expected to be a factor of three smaller and the polarisability signature of Fig. 3 accordingly clearer. This data set will also allow the extraction of $\alpha_{\pi}$ and $\beta_{\pi}$ independently, as well as the determination of the quadrupole polarisability $\alpha_{2}-\beta_{2}$. In addition, the first value for the kaon polarisability is in reach, using the kaons in the beam that are tagged by Cerenkov (CEDAR) detectors, and employing the same analysis technique as for the pion.

\section{Chiral dynamics in Primakoff pion production processes}

Along with refining the analysis described above, further processes on chiral dynamics in reach with the same COMPASS data were investigated [4]. Since long, the chiral anomaly in the process $\pi^{-} \gamma \rightarrow \pi^{-} \pi^{0}$ is of interest, however the analysis of this channel is still underway.

The detailed study of two-pion production at low energy is also not yet finished for the neutralpion case $\pi^{-} \gamma \rightarrow \pi^{-} \pi^{0} \pi^{0}$. For the charged case $\pi^{-} \gamma \rightarrow \pi^{-} \pi^{-} \pi^{+}$, however, the analysis [21] of 
the data from the pilot run in the year 2004 has been completed, and is discussed in the following subsection.

\section{1 $\pi^{-} \gamma \rightarrow \pi^{-} \pi^{-} \pi^{+}$process at low final-state mass}

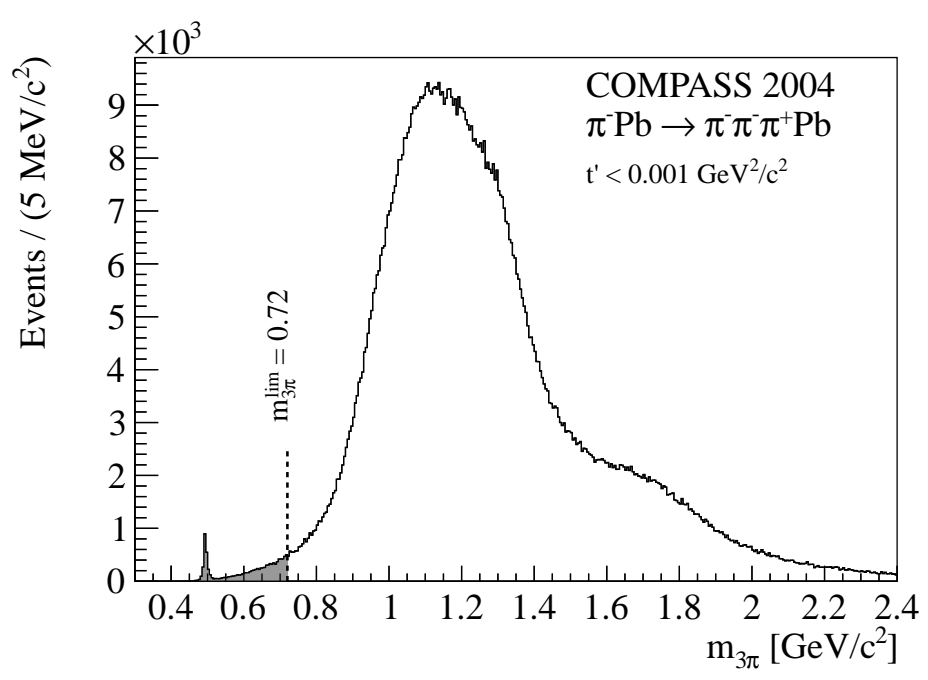

Figure 7: Mass spectrum for the reaction $\pi^{-} \mathrm{Pb} \rightarrow \pi^{-} \pi^{-} \pi^{+} \mathrm{Pb}$ in the Primakoff region $Q^{2}=t^{\prime}<$ $0.001 \mathrm{GeV}^{2} / c^{2}$. Events from the decay of the kaons in the incident beam are seen as a peak at $m_{3 \pi}=m_{K^{-}} \approx$ $0.49 \mathrm{GeV}^{2} / c^{2}$. The region of interest for chiral dynamics up to $m_{3 \pi}=5 m_{\pi} \approx 0.72 \mathrm{GeV}^{2} / c^{2}$ is highlighted in grey. Figure from [22].

The three charged-pion final state mass spectrum obtained in Primakoff kinematics off lead nuclei is shown in Fig. 7. While the region above about $1 \mathrm{GeV}$ exhibits the usual shape including three-pion resonances discussed in the next subsection, the low-mass tail is of specific interest in terms of chiral dynamics: Since the tails of known resonances play only a minor role, and the relative pion momenta are low, the kinematics lies in the region where ChPT is applicable. ChPT provides predictions for the absolute cross section to leading [6] and next-to-leading [23] order. In order to compare on the absolute level, a flux normalization for the data has to be determined. Here, the kaon component of the hadron beam is used, since some of the kaons decay in the free space around the target with well-known branching into the same three-pion final state as under study here, also with small (rather vanishing) momentum transfer. Dividing by the fraction of kaons to pions in the beam, the effective flux of pions is deduced from the observed kaon decays. Measuring the same final state, it features obviously a similar reconstruction efficiency, that has to be propagated to the full mass spectrum of interest only moderately by the Monte Carlo simulation of the setup.

The result is published in [22]. The absolute cross-section has been determined in five bins of the final-state mass from threshold at $3 m_{\pi}$ up to $5 m_{\pi}$. The data agree with the expectation from tree-level ChPT on the level of the experimental uncertainty of $20 \%$. This confirms, on the one hand, the extension of the ChPT approach for processes involving the coupling of four pions, i.e. the leading order, to processes involving the additional coupling to a photon. On the other hand, it demonstrates that the Primakoff technique in the form of Eq. 2.1 can be safely employed. In terms 
of studying ChPT, the neutral channel $\pi^{-} \gamma \rightarrow \pi^{-} \pi^{0} \pi^{0}$ will be of higher relevance, since for this channel higher-order loop corrections are expected to play a larger role [23]. The analysis of the corresponding data is underway.

For determining the intensity of the $\pi \gamma \rightarrow \pi \pi \pi$ process in the form expected from ChPT, the data in the low-mass range of interest have been fitted. Technically, this was done by including an amplitude with the five-dimensional functional form given by ChPT in a partial-wave analysis (PWA), where the intensities of the partial waves are free parameters [22]. Such PWA methods had been developed and used for the determination of the contributions from resonances with specific $J^{P C}$ quantum numbers, as discussed in the following.

\subsection{Radiative coupling of resonances in $\pi^{-} \gamma \rightarrow \pi^{-} \pi^{-} \pi^{+}$}

COMPASS has many opportunities to study meson resonance properties. In the invariant-mass spectrum obtained for the final state $\pi^{-} \pi^{-} \pi^{+}$from pions scattering off nuclei at very low momentum transfer $t^{\prime}<0.001 \mathrm{GeV}^{2} / c^{2}$, the well-known resonances $a_{1}(1260), a_{2}(1320)$ and $\pi_{2}(1670)$ contribute. For understanding their quantum numbers and the involved production mechanisms, a partial-wave analysis has been performed [24].

The $a_{2}$ resonance appears at small $t^{\prime}$, which is special in two ways: First, in strong production via Pomeron exchange the expected $t^{\prime}$ dependence is $t^{\prime} \exp \left(-b t^{\prime}\right)$ with $b \approx 400(\mathrm{GeV} / \mathrm{c})^{-2}$ [22], in contrast to the observed peak at smallest values. This shows that the resonance is not produced via the strong interaction, but rather by photon exchange following 2.1, which also approaches 0 as $t^{\prime} \rightarrow 0$, however on the unobservably small scale of the peak structure. Secondly, the data allow for determining the phase between the two resonances as explained above, and by this the change of production mechanism for the $a_{2}$ from electromagnetic to strong production becomes explicit: While the difference between the (real) production of the $a_{2}$ via photon exchange and the (imaginary) production of the $a_{1}$ via Pomeron exchange leads to the phase difference in the range of $-90^{\circ}$ for smallest momentum transfers, this difference vanishes at higher momentum transfer, when both production mechanisms turn to strong interaction.

Having ensured that the $a_{2}$ is produced via $\pi \gamma$ coupling, the measurement of the absolute cross-section for this process allows the determination of the radiative coupling of the resonance. The required normalization is obtained analogously to the kaon-decay procedure described above, using the Monte Carlo simulation in order to extrapolate the acceptance from the kaon mass to the resonance mass. COMPASS has observed with the same method the radiative coupling of the $\pi_{2}$ resonance. The respective radiative widths, $\Gamma_{0}\left(a_{2}(1320) \rightarrow \pi \gamma\right)=\left(358 \pm 6_{\text {stat }} \pm 42_{\text {syst }}\right) \mathrm{keV}$ and $\Gamma_{0}\left(\pi_{2}(1670) \rightarrow \pi \gamma\right)=\left(118 \pm 11_{\text {stat }} \pm 27_{\text {syst }}\right) \mathrm{keV}$, are published in [24].

\section{Summary}

In summary, several reactions for testing the chiral dynamics, that are accessible with the COMPASS experiment at CERN, have been discussed. The measurement of the pion polarisability reveals a value in agreement with the prediction of ChPT, and in contradiction to previous measurements. In the process $\pi \gamma \rightarrow 3 \pi$, chiral dynamics at low relative momenta could be observed. At higher energies, it was demonstrated that COMPASS can determine radiative couplings of meson resonances with unprecedented precision. 


\section{References}

[1] C. Adolph et al. [COMPASS Collaboration], Phys. Rev. Lett. 114, 062002 (2015).

[2] G. Ecker, these proceedings.

[3] H. Primakoff, Phys. Rev. 81, 899 (1951).

[4] N. Kaiser and J. M. Friedrich, Eur. Phys. J. A36, 181 (2008).

[5] Yu. M. Antipov et al., Phys. Lett. B121, 445 (1983).

[6] N. Kaiser and J. M. Friedrich, Eur. Phys. J. A39, 71 (2009).

[7] N. Kaiser and J. M. Friedrich, Nucl. Phys. A812, 186 (2008).

[8] N. Kaiser, Nucl. Phys. A837, 87 (2010).

[9] J. M. Friedrich, Habilitation treatise, TU München 2012, CERN-THESIS-2012-333, http://cds.cern.ch/record/1566653.

[10] J. Ahrens et al., Eur. Phys. J. A23, 113 (2005).

[11] D. Babusci et al., Phys. Lett. B277, 158 (1992).

[12] J. F. Donoghue and B. R. Holstein Phys. Rev. D48, 137 (1993).

[13] A. E. Kaloshin and V. V. Serebryakov, Z. Phys. C64, 689 (1994).

[14] L. V. Fil'kov and V. L. Kashevarov, Phys. Rev. C73, 035210 (2006).

[15] B. Pasquini, D. Drechsel and S. Scherer, PoS of “Chiral Dynamics 09”, Bern, 037 (2009).

[16] B. Pasquini, D. Drechsel and S. Scherer, hys. Rev. C 77, 065211 (2008) and Phys. Rev. C81, 029802 (2010).

[17] B. Pasquini, J. M. Friedrich, priv. comm.

[18] J. Gasser, M. A. Ivanov and M. E. Sainio, Nucl. Phys. B745, 84 (2006).

[19] J. Beringer et al. [Particle Data Group], Phys. Rev. D86, 010001 (2012).

[20] T. Nagel, PhD thesis, Tech. Univ. München (2012), CERN-THESIS-2012-138, http://cds.cern.ch/record/1484476.

[21] S. Grabmüller, PhD thesis, Tech. Univ. München (2012), CERN-THESIS-2012-170, http://cds.cern.ch/record/1492155.

[22] C. Adolph et al. [COMPASS Collaboration], Phys. Rev. Lett. 108,192001 (2012).

[23] N. Kaiser, Nucl. Phys. A848, 198 (2010).

[24] C. Adolph et al. [COMPASS Collaboration], Eur. Phys. J. A50, 79 (2014). 